\title{
Social distancing mediated generalized model to predict epidemic spread of COVID-19
}

\author{
Kashif Ammar Yasir (10 . Wu-Ming Liu
}

Received: 29 December 2020 / Accepted: 29 March 2021 / Published online: 11 April 2021

(C) The Author(s), under exclusive licence to Springer Nature B.V. 2021

\begin{abstract}
The extensive proliferation of recent coronavirus (COVID-19), all over the world, is the outcome of social interactions through massive transportation, gatherings and population growth. To disrupt the widespread of COVID-19, a mechanism for social distancing is indispensable. Also, to predict the effectiveness and quantity of social distancing for a particular social network, with a certain contagion, a generalized model is needed. In this manuscript, we propose a social distancing mediated generalized model to predict the pandemic spread of COVID-19. By considering growth rate as a temporal harmonic function damped with social distancing in generalized Richard model and by using the data of confirmed COVID-19 cases in China, USA and India, we find that, with time, the cumulative spread grows more rapidly due to weak social distancing as compared to the stronger social dis-
\end{abstract}

K. A. Yasir $(\varangle)$

Department of Physics, Zhejiang Normal University, Jinhua 321004, China

e-mail: kashif_ammar@yahoo.com

W. Liu

Beijing National Laboratory for Condensed Matter Physics, Institute of Physics, Chinese Academy of Sciences, Beijing 100190, China

W. Liu

School of Physical Sciences, University of Chinese Academy of Sciences, Beijing 100190, China

W. Liu

Songshan Lake Materials Laboratory, Dongguan 523808,

Guangdong, China

e-mail: wliu@iphy.ac.cn tancing, where it is explicitly decreasing. Furthermore, we predict the possible outcomes with various social distancing scenarios by considering highest growth rate as an initial state, and illustrate that the increase in social distancing tremendously decreases growth rate, even it tends to reach zero in lockdown regimes. Our findings not only provide epidemic growth scenarios as a function of social distancing but also provide a modified growth model to predict controlled information flow in any network.

Keywords COVID-19 - Growth models - Epidemiology $\cdot$ Network formation and growth

\section{Introduction}

The prevention from coronavirus (COVID-19), all over the world, has become an utmost challenge for mankind [1-6]. It has cost hundreds of thousands of lives and affected millions, and still growing with its ruthless nature [7]. In 14th century, the bubonic Black Death took nine years to proliferate across Europe because it was restricted by the average $1.5 \mathrm{~km}$ daily travel dispersion of that time [8]. However, the recent COVID-19 has no such limitations and it just took days to spread all over the world. The reason behind such frightening difference is in the increase in social interactions stimulated by massive transportation networks, gatherings and overall population growth, which led to an aggressive spread of COVID-19[6,9,10]. The only way 
to minimize social interactions is to implement social distancing mechanism, or policies, and, more importantly, closing or decreasing mobility in transportation networks-especially in social networks where the contagion factor is higher [11-14].

Over the years, the research on network epidemiology [15] has enabled us to analyze the growth of pandemic processes by developing a wide range of numerical methods and models [16-19]. These models notably include SIR [20], SEIR [21], and neural network-based control models [22], which are validated for the analysis and short-term prediction of various previous [23-26] and recent pandemics, like COVID-19 [27-32]. However, the mechanistic models are quite fragile because of the dependence on underlying spatio-temporal microscopic processes, which are often missing in ongoing metapopulation pandemics $[33,34]$.

Meanwhile, the phenomenological processes-which are more robust in calibration with empirical data [35] - like exponential, generalized exponential, logistic and Richard growth models have been validated to provide the dynamical analysis of the pandemic, like Black Death, Ebola virus, influenza, Zika virus, SARS, MERS [36-39], even for COVID-19 [40,41]. Among them, generalized logistic and Richard models, in spite of crucial dependence on enough empirical data, provide more reliable analysis than exponential models, because of the dynamical saturation at the later stages of pandemic $[42,43]$. Although, the phenomenological models have been sufficiently tested, but there is an absence of a standardized model to incorporate the element of social distancing with respect to the growth rate. Thus, to successfully design, apply and monitor social distancing mechanism, an effective as well as generalized mathematical model is needed to predict the usefulness of the social distancing for local (national) or non-local (global) in less or more contagious social networks.

In this manuscript, we proposed a social distancing induced generalized model to predict the growth of COVID-19. By modeling daily growth rate as a time dependent harmonic function, which is damped with the distance between each entity and its surrounding entities in a social network, we study the effects of social distancing (or lockdown) on the cumulative growth of the spread. We illustrate the comparison between state of the art growth mathematical models with and without modified growth rate and find that the modified Richard model, with harmonically modified growth rate, provides the most reliable growth predictions. By using modified Richard model, in calibration with empirical data of China, USA and India, we show that the daily growth rate as well as the total cumulative number of confirmed cases are (or could) increasing at weak social distancing factor in each network. However, the higher values of social distancing are significantly reducing the growth rate, even can be reduced to zero in lockdown regime.

\section{Social Distancing Mediated Growth Model.}

The phenomenological models mainly consist of generalized growth $(\mathrm{GG})$ model $\dot{N}_{c}(t)=r N_{c}(t)^{p}$, which at $p=1$ yields into simple exponential growth (EG) model, generalized Richard growth (GRG) model $\dot{N}_{c}(t)=r N_{c}(t)^{p}\left(1-N_{c}(t)^{\alpha} / K^{\alpha}\right)$, which at $\alpha=1$ converts to generalized logistic growth (GLG) model and further at $p=1$ yields in classical logistic growth (CLM) model $[42,43]$. Here, () corresponds to the time derivative and $N_{c}(t)$ is the cumulative number of confirmed cases in any network at time $t . p \in[0,1]$ accounts for the exponential profile for growth trajectory, which will be constant at $p=0$, sub-exponential at $0<p<1$ and exponential at $p=1$. $K$ accounts for the total asymptotic capacity, or total number of nodes in any social network. $\alpha$ is a scaling parameter and accounts for any asymmetric deviation of logistic trajectories. In all these models, the most crucial parameter is $r$, which defines the growth rate or the number of infections per day.

The growth rate $r$, or the quantity of spread (flow) per unit time, is the main ingredient to measure the cumulative amount of spread in any network, irrespective of mechanistic approach or phenomenological methods. In all of the previous models that are designed to measure the collective magnitude of the spread, the growth rate is used as a constant parameter. But, in actual, growth rate is the factor that varies with time depending upon the underlying microscopic network parameters, especially for meta-populated contagious network where epidemic spread is needed to be measured with respect to social distancing. Further, in accordance with the empirical data, daily growth rate $r$ mostly follows time dependent nonlinear trends rather than linear trajectories. Therefore, in order to incorporate time (daily) fluctuation in growth rate, we consider $r$ as a 
Table 1 Social distancing parameter $L_{D}$ categorization

\begin{tabular}{|c|c|c|c|}
\hline Stages & Parametric domains for $L_{D}$ & Subdomains & Policy description \\
\hline \multirow[t]{2}{*}{ Self-induced social distancing } & \multirow[t]{2}{*}{$+1 \geq L_{D} \geq 0$} & $L_{D}=+1$ & $\begin{array}{l}\text { Zero distance between network nodes leading to max- } \\
\text { imum spread scenario. }\end{array}$ \\
\hline & & $+1>L_{D} \geq 0$ & $\begin{array}{l}\text { Self-induced social distancing protocols exist but } \\
\text { there is no force confinement. The level of adapta- } \\
\text { tion of protocols by individuals corresponds to } L_{D} \\
\text { variation between }+1 \text { and } 0 \text {. }\end{array}$ \\
\hline \multirow[t]{2}{*}{ Forced confinement (Lockdown) } & \multirow[t]{2}{*}{$0>L_{D} \geq-1$} & $0>L_{D}>-1$ & $\begin{array}{l}\text { Forced Confinement (lockdown) scenario where the } \\
\text { social interactions are prohibited. The strength of } \\
\text { forced confinement corresponds to the variation of } L_{D} \\
\text { from } 0 \text { and }-1 \text {. }\end{array}$ \\
\hline & & $L_{D}=-1$ & $\begin{array}{l}\text { Extreme lockdown (quarantine) scenario where the } \\
\text { network nodes are so distant from one another that } \\
\text { infection cannot spread. }\end{array}$ \\
\hline
\end{tabular}

The categorization of $L_{D}$ is based on the potential social distancing and forced confinement factors, like social gatherings, social interactions in transportation and educational institutes. It will be based on the policies adopted by the governments

time dependent harmonic function, which is damped or perturbed over the distance between any node and its neighboring node(s),

$$
\begin{aligned}
\frac{d^{2} r(t)}{d t^{2}} & =l_{1}^{2} r_{1}(t)+l_{2}^{2} r_{2}(t)+l_{3}^{2} r_{3}(t) \ldots l_{K}^{2} r_{K}(t) \\
& =\sum_{i=1}^{K} l_{i}^{2} r_{i}(t)=L_{D}^{2} r(t)
\end{aligned}
$$

where, $K$ is the total number of nodes (entities) in the network. $r(t)$ is total growth rate of the system defined over the transmission rate of each entity of network to its neighboring entities $r_{i}(r), i \rightarrow[1,2,3, \ldots, K]$. Similarly, $L_{D}$ is the effective distance between the network nodes, which is defined over the distance (or the strength of social interactions) of each entity in the network with its surrounding entities $l_{i}, i \rightarrow$ $[1,2,3, \ldots, K]$.

We named $L_{D} \in[-1,1]$ as social distancing parameter, the value of which can be ranged from -1 to 1 , as described in Table 1 . Here $L_{D}=1$ corresponds to the zero distance between nodes leading to maximum spread scenario. $1>L_{D}>0$ means that there is social distancing but still the infection can be transferred. It can be related to a situation where protocols for social distancing are present but there is no lockdown. $0>L_{D}>-1$ represents a scenario of lockdown, means there is a considerable distance between each entity. In this case, the spread rate, instead of increasing, will decrease to zero from the initial infections state $N_{c}(0)=N_{0}$. Finally, $L_{D}=-1$ corresponds to the quarantine situation and there is no chance of social interaction. Both $L_{D}= \pm 1$ are ideal scenarios and in analogy, one can consider that there is a video which is going to be viral on internet and we want to stop it. Here $L_{D}=1$ means, we by ourselves share to all end-users and for $L_{D}=-1$ means, we immediately shutdown the whole internet.

We consider the particular solution for second-order harmonic differential equation for $r(t)$, which yield in a hyperbolic function $r(t)=r_{0} \sinh \left(L_{D} t\right)$, where $r(0)=r_{0}$ is the initial growth rate at $t=0$. With perturbed $r(t)$, the modified generalized growth (MGG) model will be read as $N_{c}(t)=r_{0} \sinh \left(L_{D} t\right) N_{c}(t)^{p}$, which, similarly at $p=1$ yields in modified exponential growth (MEG) model. The modified Richard growth (MRG) model can be expressed as,

$\frac{d N_{c}(t)}{d t}=r_{0} \sinh \left(L_{D} t\right) N_{c}(t)^{p}\left(1-\left(\frac{N_{c}(t)}{K}\right)^{\alpha}\right)$,

which again at $\alpha=1$ yields in modified logistic growth (MLG) model.

\section{Conventional phenomenological models and modified growth model}

The comparison between EG, GG, GLG, GRG and MRG models is illustrated in Fig. 1a and comparison between modified models (MEG, MGG, MLG and MRG) is presented in Fig. 1b, by using data of cumulative COVID-19 growth in China as an example. 


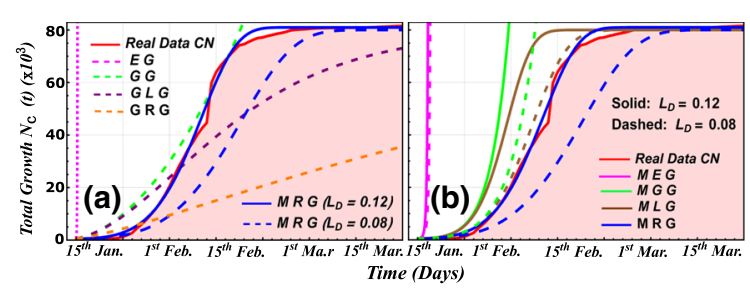

Fig. 1 Conventional models in comparison with modified Richard model. a The simulated of cumulative growth $N_{c}(t)$ versus time for comparison between EG (magenta), GG (green), GLG (purple), GRG (orange) and MRG (blue) models with respect to the data of real confirmed COVID-19 cases in China $(\mathrm{CN})$, red curve. It can be seen that the MRG model shows more accurate fit to the real data at given parameters. b The comparison between cumulative growth $N_{c}(t)$ modified models (MEG (magenta), MGG (green), MLG (brown) and MRG (blue)) with respect to the real COVID-19 data of China, red curve. Here $L_{D}=0.12$ and $=0.08$ are for solid and dashed curves, respectively. It is illustrated that, even with modified daily growth rate $r(t)$, the MRG model shows more compelling results with respect to COVID-19 growth of China. The remaining parameters are $r_{0}=18 /$ day with $N_{c}(0)=N_{0}=500, p=0.48, \alpha=0.22$ and $K=81000$ (with respect of COVID-19 data of China)

(Note: The real COVID-19 data used in the manuscript was adopted from Refs. [1-5], till August 8, 2020.) It can be clearly seen that EG model follows a rapid exponential growth (magenta curve), as expected, and GG model follows a (sort-of) sub-exponential growth (green curve) because of $p$, see Fig. 1a. On the other hand, both GLG (purple curve) and GRG (orange curve) models follow delayed cumulative growth patterns, but still they are incomparable with the actual growth in China at given parameters. However, MRG model (blue curve), at $L_{D}=0.12$ shows almost perfect regressive growth patterns with respect to COVID-19 data of China, which, at slightly higher social distancing parameter $L_{D}=0.08$ (blue dashed curve), shows notably delayed growth to the saturated state.

Similarly, in the comparison between modified models (MEG, MGG, MLG and MRG), one can clearly note that the MRG model still provides a more reliable fit to the real data at given parameters, see Fig. 1 b. The interesting thing is that $L_{D}$ not only delays the growth of MRG model, but it also reduces the speed of aggression in all modified models, which further supports our concept of social distancing induced delay in phenomenological model.

The both $L_{D}$ and $r_{0}$ are crucial for the calibration of modified growth models, as illustrated in Fig. 2, where the detailed dynamics of MRG, as a function of $L_{D}$

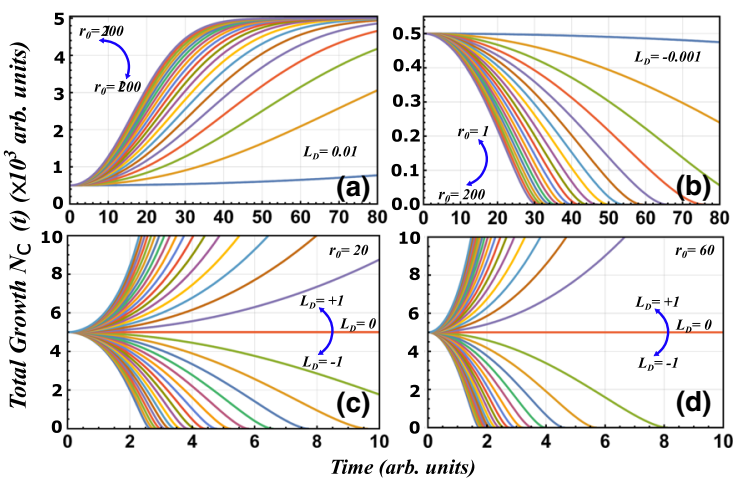

Fig. 2 The dynamics of MRG model with respect to social distancing parameter $L_{D}$ and initial daily growth rate $r_{0}$. a and b are $N_{c}(t)$ versus time for various $r_{0}$ (from 1 to 200), at $L_{D}=0.01$ and $=-0.001$, respectively, where the total network size $K=5000$ and initial number of confirmed patients $N_{0}=500$. It is illustrated that the cumulative growth $N_{c}(t)$, in both social distancing $L_{D}>0$ and forced confinement domain $L_{D}<0$, crucially depends on the initial daily rate $r_{0}$. The higher initial rate $r_{0}$ causes faster growth (decay) of $N_{c}(t)$ in $L_{D}>0$ $\left(L_{D}<0\right)$ regimes. c) and $\left(\mathbf{d}\right.$ are $N_{c}(t)$ versus time for $L_{D}$ ranging from -1 to 1 , at $r_{0}=20$ and $r_{0}=-60$, respectively, where $K=10000$ and $N_{0}=5000$. It further strengthens the argument of (a) and (b), regarding the effects of initial rate $r_{0}$ on $N_{c}(t)$ in presence of $L_{D}$. Remaining parameters used in numerical calculations are same as in Fig. 1

and $r_{0}$, versus time are shown. In a high social distancing scenario (i.e. $L_{D}=0.01$ ), the cumulative number $N_{c}(t)$ (or total infections) moves from rapid growth to delayed, or slow, growth trends with higher to lower initial growth rate $1 \leq r_{0} \leq 200$, respectively (see Fig. 2a), which is obvious. However, in lockdown regime (i.e. $\left.L_{D}=-0.001\right)$, the $N_{c}(t)$ will decrease from initial state, which is $N_{0}=500$, to the zero infection scenario, as can be seen in Fig. 2b. The interesting thing is that it will decay faster at higher $r_{0}$ as compared to low values of $r_{0}$. It is because the $N_{c}(t)$ will follow same initial rate to decay as it is following in growth scenario. It can be understood through an example that if we are driving a car at high speed and we suddenly apply breaks, then we will feel more deceleration as compared to the case when we apply breaks at a lower speed. More detailed behavior of $N_{c}(t)$, under influence of $L_{D}$, can be seen in Fig. 2c and d, with $r_{0}=20$ and $r_{0}=60$, respectively. The $N_{c}(t)$ transforms from fast growth regime to fast decay regime by changing $L_{D}$ from +1 to -1 , which get more rapid with increase in $r_{0}$, see Fig. $2 d$. It reveals that in growth regime (i.e. $\left.1 \geq L_{D}>0\right), N_{c}(t)$ will reach sooner to the maxi- 
mum network capacity $K$ at higher values of $L_{D}$ and in decay regime (i.e. $\left.0>L_{D} \geq-1\right), N_{c}(t)$ will decay sooner to zero infection state from the initial number of infections, at lower values of $L_{D}$. At $L_{D}=0, N_{c}(t)$ will not be modified and will remain equivalent to the initial number of infections $N_{0}$.

\section{Robust influence of social distancing on cumulative growth of COVID-19.}

The influence of social distancing $L_{D}$ is illustrated in Fig. 3, where the multiple outcomes of $N_{c}(t)$ and $r(t)$ are predicted with various calibrations for $L_{D}$, with respect to the real COVID-19 data of China, USA and India. With a small increase in $L_{D}, N_{c}(t)$ grows rapidly and by strengthening $L_{D}$ the ratio of growth $N_{c}(t)$ decreases, as illustrated in Fig. reffig $3 \mathrm{a}$, $\mathrm{b}$ and $\mathrm{c}$. The blue curves (at $L_{D}=0.12$ for China, $L_{D}=0.61 \times 10^{-3}$ for USA, and $L_{D}=0.022$ for India) indicate the growth of cumulative number of infections matching with the real data. But when we slightly increase or decrease social distancing parameter $L_{D}$, the cumulative growth $N_{c}(t)$ gets significantly enhanced (rapid) or damped (delayed), respectively, at a fixed arbitrary network size $K$. These modifications in $N_{c}(t)$ can also be validated from the spectrum of $r(t)$, shown in Figs. 3d, e and f, where blue curves provide almost a perfect fit to the real growth rate of infections. By changing conditions for social distancing, the $r(t)$ will either reach rapidly or slowly to the maximum network size with increase or decrease in $L_{D}$, providing the evidence for the reliability of our model.

It should be noted that the narrow and wide time spans, or the so-called standard deviation, correspond to the faster and slower approach to the maximum network size, which we considered fix and arbitrary (except for China because we have the state of total infections) in order to discuss rapid growth patterns. But it can be changed with the real social/sub-social network sizes. Further, one can question the notable variations in the quantitative values of $L_{D}$ for network of China, USA, and India. It is not only because of the different network sizes $K$, but it is also because of crucial role of $r_{0}$. With higher $r_{0}$ and $K$, a small change in $L_{D}$ will notably affect the growth trajectory, but for smaller $r_{0}$ and $K$, a strong change in $L_{D}$ will be needed in order to produce similar influences. In this way, our model provides more flexibil- ity to map and predict real-time trajectories, see supplementary materials [44] for details. Not only this, but also by observing different trends for $N_{c}(t)$, with respect to $L_{D}$, one can predict the future growth patterns with change in current social distancing scenarios. For example, a slight increase in $L_{D}=0.61 \times 10^{-3}$ to $1.5 \times 10^{-3}$ and $L_{D}=0.022$ to 0.031 , will increase the daily rate $r(t) \approx 80 \times 10^{3} /$ day to $120 \times 10^{3} /$ day and $r(t) \approx 100 \times 10^{3} /$ day to $130 \times 10^{3} /$ day for USA and India, respectively. Similarly, a small decrease in $L_{D}$ yields in significant decrease in $r(t)$, as illustrated in Fig. 3d, e and f.

\section{Predicting outcomes of COVID-19: Daily growth rate in forced confinement scenarios}

The rapid diffusion in the growth spectrum arrives in lockdown regime, $L_{D}<0$, as illustrated in Fig. 4, where we used MRG model to predict spectrum of outcomes for $r(t)$ versus various $L_{D} \in[-1,1]$, by considering the peak value as an initial state. In the case of China, peak value of $r(t)$ (that we consider) occurs around $5^{\text {th }}$ February. At that peak rate, which is around $r(t)=4000 /$ day, the MRG model, at $L_{D}=-0.007$, exactly follows the decay trend of COVID-19 in China, which illustrates the effectiveness of strict lockdown policies of China. Although, ideal scenarios, i.e. $L_{D}= \pm 1$ are realistically impossible, but it can be seen that the scenario for COVID-19 dynamics in China could have been worse (or better) with relaxation (or increase) in lockdown and social distancing policies. For example, if $L_{D}$ was varied from -0.007 to -0.001 , the COVID-19 growth tends could have extended from $\approx 25^{\text {th }}$ February to $\approx 5^{\text {th }}$ April, even in the lockdown domain. Further increase in $L_{D}$, in $L_{D}>0$ regime, could have led the country to worse scenarios, as illustrated in Fig. 4a.

In the case of USA, so-far, the maximum $r(t) \approx$ $80000 /$ day is occurring around $\approx 20$ th July, which can decay to zero around 15th August if it follows the decay trajectory at $L_{D}=-0.001$. But it can be extended to 1 st November with an increase in $L_{D}$ to -0.0001 , or it can continue to increase in less social distancing regime, i.e. $L_{D}>0$, as can be seen in Fig. 4b. Similarly, for India, where the growth rate $r(t)$ is still increasing, the maximum $r(t) \approx 650000 /$ day till 8th August can tend to decay with $L_{D}<0$, and can continue rapid increase with $L_{D}>0$, in spite imple- 

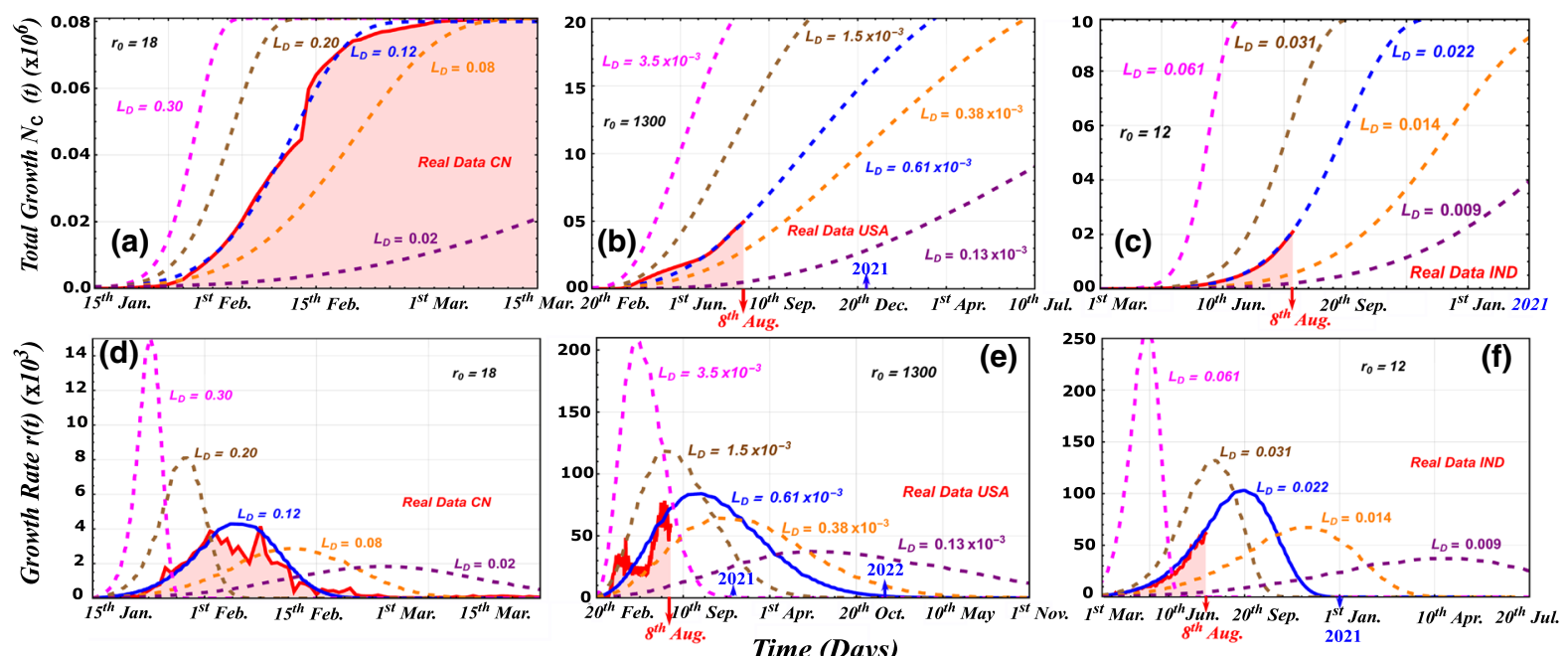

Fig. 3 Social distancing induced robust decrease in COVID19 cumulative growth. The MRG model versus various $L_{D}$, for growth $N_{c}(t),(\mathbf{a}),(\mathbf{b})$ and $(\mathbf{c})$, and growth rate $r(t),(\mathbf{d})$, (e), and (f), with respect to the real COVID-19 data of confirmed cases in China (CN), USA and India (IND), respectively. The real data of COVID-19 is represented by the red curves. Magenta, brown, blue, orange, and purple curves correspond to $L_{D}=0.3,0.2,0.12,0.08$ and 0.02 in (a) and (d), $L_{D}=3.5 \times 10^{-3}, 1.5 \times 10^{-3}, 0.61 \times 10^{-3}, 0.38 \times 10^{-3}$ and $0.13 \times 10^{-3}$ in (b) and (e), and $L_{D}=0.061,0.031,0.022,0.014$ and 0.009 in (c) and (f), respectively. $r_{0}=18$ and $K=81000$ for (a) and (d), $r_{0}=1300$ and $K=25 \times 10^{6}$ for (b) and (e), and $r_{0}=12$ and $K=10 \times 10^{6}$ for (c) and (f). It can be observed that at $L_{D}=0.12 \mathrm{MRG}$ model almost exactly follows COVID-19 growth in China at particular initial rate $r_{0}$. But in the case of COVID-19 in India and USA, the MRG model, so-far, is showing fit to real at lower values of $L_{D}$ but initial rates $r_{0}$ are very high, as compared to the case of China, yielding in rapid growth of COVID-19. Thus to deal with the rapid growth of COVID-19, especially in the case of USA, a more effective policies for social distancing are needed. The other parameters used in calculations are same as illustrated in Fig. 1
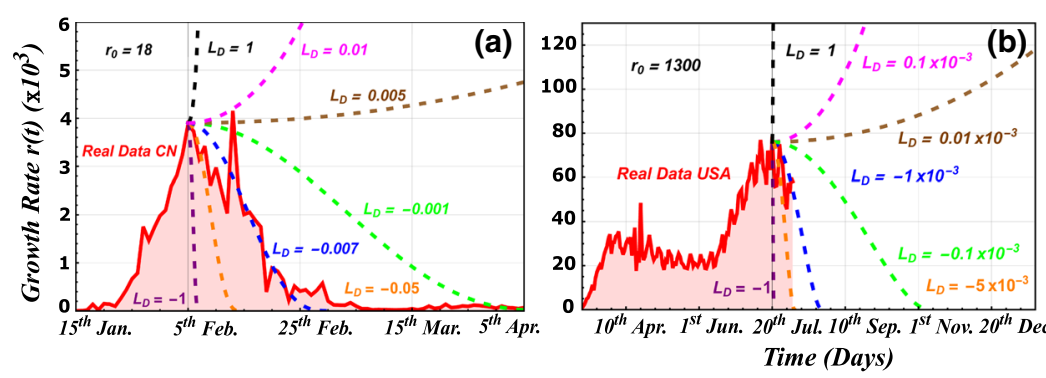

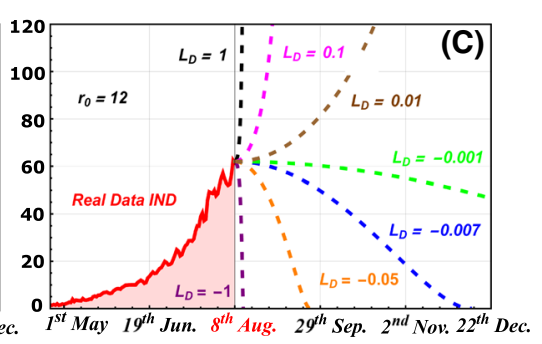

Fig. 4 Predicting the outcomes of COVID-19 daily growth rate $r_{0}$ in forced confinement domain. The growth rate $r(t)$ versus time with possible outcomes of MRG model from the state of maximum daily growth rate of COVID-19 confirm cases in China (a), USA (b), and India (c) in social distancing $L_{D}>0$ and forced confinement domain $L_{D}<0$. Here $N_{0}=4000$ with $K=82000$ for (a), $N_{0}=80000$ with $K=25 \times 10^{6}$ for $(\mathbf{b})$ and $N_{0}=65000$ with $K=10 \times 10^{6}$ for $(\mathbf{c})$. The red curves correspond to real COVID-19 data. Black, magenta, brown, green, blue, orange, and purple curves correspond to $L_{D}=$
$1,0.01,0.005,-0.001,-0.007,-0.05$ and -1 in $(\mathbf{a}), L_{D}=$ $1,0.1 \times 10^{-3}, 0.01 \times 10^{-3},-0.1 \times 10^{-3},-1 \times 10^{-3},-5 \times 10^{-3}$ and -1 in (b), and $L_{D}=1,0.1,0.01,-0.001,-0.007,-0.05$ and -1 in (c), respectively. It is observed that the increase in $L_{D}$ towards zero, or decrease in forced confinement policies, leads to the more rapid growth of daily COVID-19 cases, while decrease in $L_{D}$, or more strict force confinement, leads to the robust decay in daily COVID-19 cases. Remaining parameters used in numerical calculation are same as in Fig. 1 
mentation of social distancing. In lockdown regime, at $L_{D}=-0.05$, the infection growth rate can be zero around 20th September, but with a slight relaxation in lockdown with a small increase in $L_{D}=-0.007$ can prolong the spread till 22th December, see Fig. 4c. Further increase in $L_{D}$ can yield in rapid and frightening spread of COVID-19. Thus, to stop the spread, policies with higher social distancing factor $L_{D}$ are needed.

\section{Conclusion}

To conclude, we propose a social distancing mediated generalized model to predict the spread of COVID-19, with respect to social distancing and lockdown regimes. By considering growth rate as a time variant function of social distancing in generalized Richard model, we illustrate different possible outcomes of cumulative growth (total number of infections) and growth rate (infection per day) in different social distancing and lockdown scenarios. In weak distancing regime $\left(1>L_{D}>0\right)$, the cumulative growth as well as growth rate appears to be delayed with $L_{D}>0$, but it will still be increasing. However, in strong distancing or in lockdown regime $\left(0>L_{D}>-1\right)$, the infection spread will not only stop but will also decay from its initial state with time. Further, by using our model, we predict different future scenarios (especially for the COVID-19 spread in USA and India) with highest growth rate as initial state and show that, in absence of strict lockdown policies, the COVID-19 pandemic spread will be worst and prolonged. Thus, in order to contain the spread, in any contagion network, strong social distancing polices are necessary. Furthermore, our model is not only effective in predicting the influence of social distancing for epidemic spreads, but it can also be validated for controlling information flow in any network.

Acknowledgements This work was supported by the National Key R\&D Program of China under grants No. 2016YFA0301500, NSFC under grants Nos. 61835013 and 61775242, Strategic Priority Research Program of the Chinese Academy of Sciences under grants Nos. XDB01020300 and XDB21030300.

Data Availability Statement The empirical COVID-19 data used in the manuscript to calibrate and validate the modified phenomenological model discussed in this manuscript was adopted and is available on the Coronavirus (COVID-19) section of Center for Disease Control and Prevention (CDC),
United States website (https://www.cdc.gov /coronavirus/2019ncov/index.html).

\section{Declarations}

Conflict of interest The authors declare no competing financial interests.

Numerical methods and codes In order to govern temporal evolution of modified Richard growth model, in calibration with the empirical data, we use the NDSolve function of Mathematica 10 programming application (https://www.wolfram.com /mathematica/) to solve equation (2) by considering given parameters in the manuscript. After generating data, we use graphical application OriginPro 9.0 (https://www.originlab. com/) to illustrate and compare computed results with the empirical data.

\section{Appendix: The influence of $r_{0}$ on the cumulative growth $N_{c}(t)$ and growth rate $r(t)$}

Figure 5 illustrates the cumulative growth $N_{c}(t)$ and growth rate $\left.r_{(} t\right)$ trends for various network sizes $K$ with

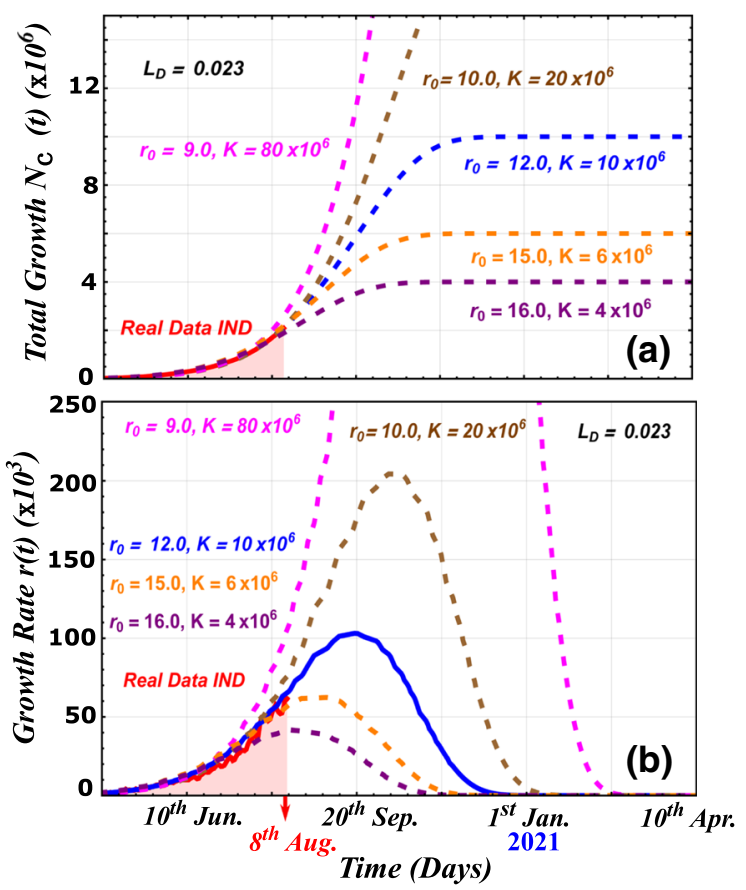

Fig. 5 (a) $N_{c}(t)$ and (b) $\left.r_{(} t\right)$ as function of time for various initial growth rates $r_{0}$ and total network size $K$, for fix social distancing $L_{D}=0.023$. Red curves correspond to real COVID19 data of India (IND), and Magenta, brown, blue, orange, and purple curves corresponds to $r_{0}=9$ with $K=80 \times 10^{6}, r_{0}=10$ with $K=20 \times 10^{6}, r_{0}=12$ with $K=10 \times 10^{6}, r_{0}=15$ with $K=6 \times 10^{6}$ and $r_{0}=16$ with $K=4 \times 10^{6}$, respectively. Remaining parameters are same as in Fig. 1 of main text 
different initial growth rates $r_{0}$, at fixed social distancing scenario $L_{D}=0.023$, by considering COVID-19 data of India. It can be seen that, with higher initial growth rate $r_{0}=16$, the network size gets shrunk to $K=4 \times 10^{6}$, and for lower rate $r_{0}=16$, the total network size increases to $K=80 \times 10^{6}$, as can be seen in Fig. 5a. It is because the growth trajectory is fixed by the applied social distancing parameter $L_{D}$, means social distancing is using same rate to stop the spread as it was initial state $r_{0}$, as can also be seen in Fig. 2 of main text. Therefore, higher $r_{0}$ will be less effective as compared to the lower rates. It can be interpreted that the social distancing $L_{D}$ is more effective for higher initial rates in comparison to lower rates. Similarly, growth rate $r(t)$ takes more time to grow and decay at lower $r_{0}$ as compared to the higher initial rate $r_{0}$, as can be seen Fig. 5b.

\section{References}

1. Coronavirus disease (COVID-19) pandemic, World Health Organization (WHO), United Nations [Online]. 2020. https://www.who.int/emergencies/diseases/ novel-coronavirus-2019 [August 8 2020]

2. Wikipedia: Coronavirus disease 2019 [Online]. 2020. https://en.wikipedia.org/wiki/Coronavirus_disease_2019 [August 8 2020]

3. Coronavirus (COVID-19), Center for Disease Control and Prevention (CDC), United States [Online]. $2020 \mathrm{https}: / /$ www.cdc.gov/coronavirus/2019-ncov/index.html [August 8 2020]

4. COVID-19, Chinese Center for Disease Control and Prevention (CCDC), P. R. of China [Online]. 2020 http://www. chinacdc.cn/en/COVID19/ [August 8 2020]

5. National Center for Disease Control (NCDC), India [Online]. 2020 https://ncdc.gov.in/ [August 8 2020]

6. Chinazzi, M., et al.: The effect of travel restrictions on the spread of the 2019 novel coronavirus (COVID-19) outbreak. Science 368, 395-400 (2020)

7. Coronavirus disease (COVID-19), dashboard, World Health Organization (WHO), United Nations [Online]. 2020. https://www.who.int/emergencies/diseases/ novel-coronavirus-2019 [August 8 2020]

8. McEvedy, C.: The bubonic plague. Sci. Am. 258, 118 (1988)

9. Anderson, R.M., Heesterbeek, H., Klinkenberg, D., Hollingsworth, T.D.: How will country-based mitigation measures influence the course of the COVID-19 epidemic? Lancet 395(10228), 931-934 (2020)

10. Thu, T.P.B., Ngoc, P.N.H., Hai, N.M., Tuan, L.A.: Effect of the social distancing measures on the spread of COVID19 in 10 highly infected countries. Sci. Total Environ. 742, 140430 (2020)

11. Ibarra-Vega, D.: Lockdown, one, two, none, or smart Modeling containing covid-19 infection. A Concept. Model. Sci. Total Environ. 730, 138917 (2020)
12. Jarvis, C.I., et al.: Quantifying the impact of physical distance measures on the transmission of COVID-19 in the UK. BMC Med. 18, 124 (2020)

13. Katz, R., Vaught, A., Simmens, S.J.: Local decision making for implementing social distancing in response to outbreaks. Publ. Health Rep. 134, 150-154 (2019)

14. Qiu, Y., Chen, X., Shi, W.: Impacts of social and economic factors on the transmission of coronavirus disease 2019 (COVID-19) in China. J. Popul. Econ. 33, 1127-1172 (2020)

15. Pastor-Satorras, R., Castellano, C., van Mieghem, P., Vespignani, A.: Epidemic processes in complex networks. Rev. Mod. Phys. 87, 925 (2015)

16. Moore, C., Newman, M.E.J.: Epidemics and percolation in small-world networks. Phys. Rev. E 61, 5678 (2000)

17. Wang, J.: J. Mathematical models for COVID-19: applications, limitations, and potentials, Public Health Emerg. 49 (2020)

18. Hamilton, K.E., Pryadko, L.P.: Tight lower bound for percolation threshold on an infinite graph. Phys. Rev. Lett. 113, 208701 (2014)

19. Karrer, B., Newman, M.E.J., Zdeborovà, L.: Percolation on sparse networks. Phys. Rev. Lett. 113, 208702 (2014)

20. Laguzet, L., Turinici, G.: Individual vaccination as nash equilibrium in a SIR model with application to the 20092010 Influenza A (H1N1) epidemic in France. Bulletin Math. Biol. 77, 1955-1984 (2015)

21. Lekone, P.E., Finkenstädt, B.F.: Statistical inference in a stochastic epidemic SEIR model with control intervention: Ebola as a case study. Biometrics 62, 1170-1177 (2006)

22. Dandekar, R., Barbastathis, G.: Neural Network aided quarantine control model estimation of global Covid-19 spread, arXiv:2004.02752v1 (2020)

23. Dye, C., Gay, N.: Modeling the SARS epidemic. Science 300(5627), 1884-1885 (2003)

24. Zhao, Z., et al.: Effect of social group dynamics on contagion. Phys. Rev. E 81, 056107 (2010)

25. Smieszek, T., et al.: Reconstructing the 2003/2004 H3N2 influenza epidemic in Switzerland with a spatially explicit, individual-based model. BMC Infec. Diseases 11, 115 (2011)

26. Gautreau, A., Barrat, A., Barthelemy, M.: Global disease spread: statistics and estimation of arrival times. J. Theor. Biol. 251, 509 (2008)

27. Chang, S. L., Harding, N., Zachreson, C., Cliff, O. M., Prokopenko, M.: Modelling transmission and control of the COVID-19 pandemic in Australia, arXiv:2003.10218 (2020)

28. Prem, K., et al.: The effect of control strategies to reduce social mixing on outcomes of the COVID-19 epidemic in Wuhan, China: a modelling study. Lancet Publ. Health 5, E261-E270 (2020)

29. Boldog, P., et al.: Risk assessment of novel coronavirus COVID-19 outbreaks outside China. J. clinic. medic. 9, 571 (2020)

30. Rocklöv, J., Sjödin, H., Wilder-Smith, A.: COVID-19 outbreak on the diamond princess cruise ship: estimating the epidemic potential and effectiveness of public health countermeasures. J. Travel Medic. 27, 3 (2020)

31. Cooper, I., Mondal, A., Antonopoulos, C.G.: A SIR model assumption for the spread of COVID-19 in different communities. Chaos, Solitons Fractals 139, 110057 (2020) 
32. Waqas,M., Farooq, M., Ahmad, R., Ahmad, A.: Analysis and Prediction of COVID-19 Pandemic in Pakistan using Time-dependent SIR Model, arXiv:2005.02353v1 (2020)

33. Sornette, D.: Predictability of catastrophic events: Material rupture, earthquakes, turbulence, financial crashes, and human birth. Proc. Natl. Acad. Sci. 99, 2522-2529 (2002)

34. Israeli, N., Goldenfeld, N.: Computational irreducibility and the predictability of complex physical systems. Phys. Rev. Lett. 92, 074105 (2004)

35. Richards, F.J.: A flexible growth function for empirical use. J. Exp. Bot. 10(2), 290-301 (1959)

36. Chowell, G.: et al., Using Phenomenological Models to Characterize Transmissibility and Forecast Patterns and Final Burden of Zika Epidemics, PLoS Curr. (2016)

37. Chowell, G.: Fitting dynamic models to epidemic outbreaks with quantified uncertainty: A Primer for parameter uncertainty, identifiability, and forecasts. Infect. Dis. Model. 2(3), 379-398 (2017)

38. Viboud, C., Simonsen, L., Chowell, G.: A generalizedgrowth model to characterize the early ascending phase of infectious disease outbreaks. Epidemics 15, 27-37 (2016)

39. Chowell, G., Tariq, A., Hyman, J.M.: A novel sub-epidemic modeling framework for short-term forecasting epidemic waves. BMC Med. 17(1), 1-8 (2019)
40. Roosa, K., et al.: Short-term Forecasts of the COVID-19 Epidemic in Guangdong and Zhejiang, China: February 1323, 2020. J Clin Med. 9(2), 596 (2020)

41. Remuzzi, A., Remuzzi, G.: COVID-19 and Italy: what next? Lancet 395(10231), 1225-1228 (2020)

42. Wu, K., Darcet, D., Wang, Q., Sornette, D.: Generalized logistic growth modeling of the COVID-19 outbreak: comparing the dynamics in the 29 provinces in China and in the rest of the world. Nonlinear Dyn (2020). https://doi.org/10. 1007/s11071-020-05862-6

43. Saif, F.: COVID-19 Pandemic in Pakistan: Stages and Recommendations, (preprint) medRxiv (2020). https://doi.org/ 10.1101/2020.05.11.20098004

44. See Supplemental Material at, web link, for the correlation between social distancing factor $L_{D}$ and initial growth rate $r_{0}$

Publisher's Note Springer Nature remains neutral with regard to jurisdictional claims in published maps and institutional affiliations. 